patients treated with both BIOs and GCs $(n=10)$. We determined baseline $(B L)$ characteristics, \% changes in BMD in the lumbar spine (LS) and total hip (TH) from $\mathrm{BL}$ to 24 months, and \% changes in serum bone turnover markers (BTMs), such as BAP, P1NP, NTX, and TRACP-5b, from BL to 6 months after dTP initiation. Dunnett's test was used for comparisons between $B(-) \mathrm{G}(-)$ and other groups.

Results: The mean ages of the $B(-) G(-), B(+) G(-), B(-) G(+)$, and $B(+) G(+)$ groups at $B L$ were $70.0,65.5,69.6$, and 71.5 years, whereas the mean duration of RA in these groups were $15.4,20.8,69.9$, and 71.5 years, respectively. Furthermore, the mean baseline DAS28-CRP levels in these groups were 2.8, 2.2, 2.8, and 2.3. The mean LS-BMD $\left(\mathrm{g} / \mathrm{cm}^{2}\right)$ at BL were $0.795,0.819,0.826$, and 0.853, whereas the mean TH-BMD at BL were $0.619,0.570,0.601$, and 0.629 , respectively. The mean $\%$ changes in LS-BMD at 24 months were $15.5 \%, 12.7 \%, 11.9 \%$, and $8.1 \%$, respectively (Fig $1 \mathrm{~A})$. There were no significant differences between $\mathrm{B}(-) \mathrm{G}(-)$ and other groups. The mean \% changes in TH-BMD at 24 months in the $B(-) G(-), B(+) G(-), B(-) G(+)$, and $B(+) G(+)$ groups were $6.4 \%, 5.3 \%, 4.4 \%$, and $1.5 \%$, respectively (Fig $1 \mathrm{~B}) \mathrm{A}$ significant difference was observed between the $B(-) G(-)$ and $B(+) G(+)$ groups $(p=0.03)$. The $\%$ changes in BTMs in the $B(-)$ $\mathrm{G}(-), \mathrm{B}(+) \mathrm{G}(-), \mathrm{B}(-) \mathrm{G}(+)$, and $\mathrm{B}(+) \mathrm{G}(+)$ groups were as follows: $\mathrm{BAP}, 90.5 \%$, $44.0 \%, 29.5 \%$, and $87.7 \%$; P1NP, $374.1 \%, 338.2 \%, 225.9 \%$, and $640.0 \%$; NTX, $75.2 \%, 106.6 \%, 42.5 \%$, and $80.5 \%$; and TRACP-5b, $75.8 \%, 43.85,20.4 \%$, and $122.3 \%$, respectively. No significant differences were observed in the changes in BTMs among the groups.
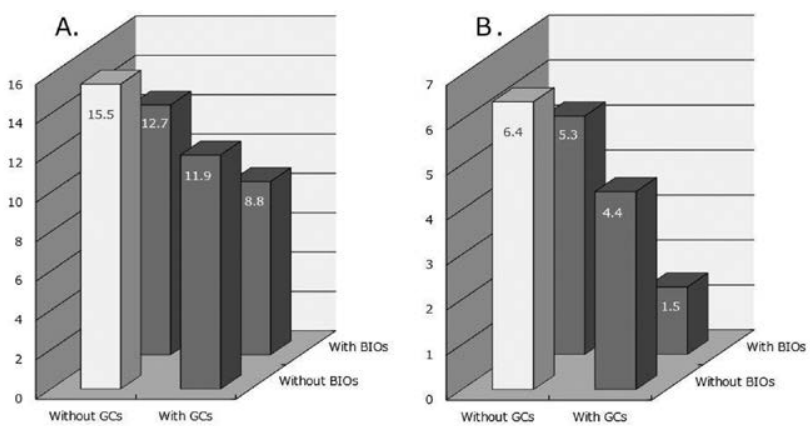

Figure 1. \% Change of Bone Mineral Density from Baseline to 24 Months A: Lumbar spine, B: Total hip

BIOs: biological agents, GCs: glucocorticoids

Conclusion: This study suggested that concomitant use of BIOs and GCs inhibited the increase in BMD induced by dTP treatment in patients with RA, particularly TH-BMD. Although BTM analysis revealed no statistical significance, GCs tended to decrease the \% change in BTMs.

References:

[1] Hirano et al. Ann Rheum Dis 2014: 73 (Suppl 2): 166

[2] Hirano et al. Ann Rheum Dis 2015: 74 (Suppl 2): 528

Disclosure of Interests: Yuji Hirano Speakers bureau: Tanabe-Mitsubishi, Pfizer, Eisai, Abbie, Chugai, Bristol-Meyers, Jansen, Astellas, UCB, Eli-Lilly, Asahikasei, Daiichi-Sankyo, Amgen, Hironobu Kosugiyama: None declared, Kyosuke Hattori: None declared, Daisuke Kihira: None declared

DOI: 10.1136/annrheumdis-2020-eular.4200

\section{AB0899 GLUCOCORTICOID-INDUCED OSTEOPOROSIS IN THE AKITA ORTHOPEDIC GROUP ON RHEUMATOID ARTHRITIS REGISTRY}

T. Kawano $^{1}$, T. Kashiwagura ${ }^{2}$, M. Kobayashi ${ }^{3}$, Y. Sugimura ${ }^{4}$, H. Sato ${ }^{5}$, N. Miyakoshi ${ }^{1}$, Y. Shimada ${ }^{1} .{ }^{1}$ Akita University Graduate School of medicine, Orthopedic, Akita, Japan; ${ }^{2}$ Akita City Hospital, Orthopedic, Akita, Japan; ${ }^{3}$ Hiraka General Hospital, Orthopedic, Yokote, Japan; ${ }^{4}$ Nakadori General Hospital, Orthopedic, Akita, Japan; ${ }^{5}$ Kita Akita Municipal Hospital, Orthopedic, Kita Akita, Japan

Background: Glucocorticoids (GC) have potent anti-inflammatory and immunosuppressive effects and are used to treat a variety of diseases. However, GC are associated with several adverse effects. Glucocorticoid-induced osteoporosis (GIO), a bone metabolism disorder, accounts for $25 \%$ of the side effects associated with GC, and long-term use of these agents leads to fragility fractures in 30 to $50 \%$ of patients [1]. GC are frequently used to treat rheumatoid arthritis (RA). No report on the current treatment status for glucocorticoid-induced osteoporosis (GIO) has been published following the publication of the new guidelines for the management and treatment of GIO issued by the Japanese Society for Bone Mineral Research provided in 2014 (Figure 1) [2].

Objectives: The present study aimed to investigate the current treatment status of GIO patients in the Akita Orthopedic Group on Rheumatoid Arthritis (AORA) registry.
Methods: This retrospective, multicenter study included 683 patients (138 men, 545 women) with fracture risk factor scores $\geq 3$ based on the new guidelines who were in the AORA registry. We examined patient characteristics, differences in patient backgrounds between treated and non-treated groups.

Results: There were no significant differences in mean GC dose between men and women $(4.0 \pm 2.3 \mathrm{mg} /$ day vs $3.6 \pm 1.8 \mathrm{mg} /$ day, $\mathrm{p}=0.08)$. The mean disease duration of RA in women was significantly longer than in men $(180.2 \pm 140.2$ months vs $143.8 \pm 129.6$ months, Untreated GIO patients were significantly more likely to be men and younger. The univariate analysis showed that clinic visits, male sex, younger age, and longer disease duration were significant risk factors for lack of therapeutic intervention for GIO. Multivariate analysis showed that being treated in a clinic, male sex, and younger age were significant risk factors for lack of therapeutic intervention for GIO.

Conclusion: Our results emphasize the importance of considering the preven tion and treatment of GIO in all patients with RA, including younger and male patients, who have lower intervention rates.

References:

[1] Weinstein RS. Clinical practice. Glucocorticoid-induced bone disease. New Engl J Med. 2011; 365(1): 62-70.

[2] Suzuki Y, Nawata H, Soen S, Fujiwara S, Nakayama H, Tanaka I, et al. Guidelines on the management and treatment of glucocorticoid-induced osteoporosis of the Japanese Society for Bone and Mineral Research: 2014 update. J Bone Miner Metab. 2014; 32(4): 337-350.

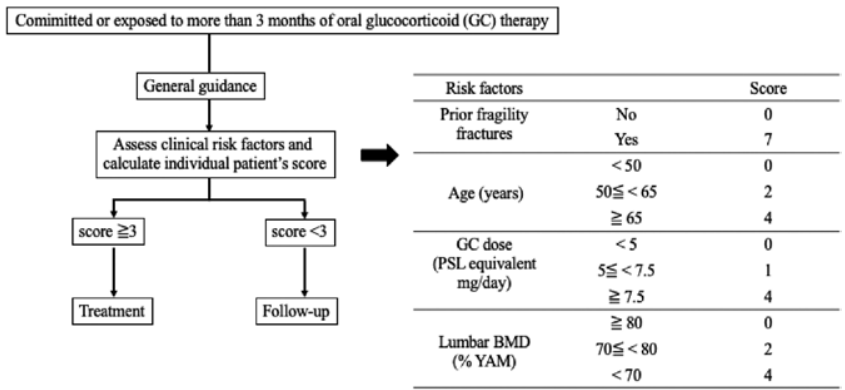

Disclosure of Interests: None declared

DOI: 10.1136/annrheumdis-2020-eular.4280

\section{AB0900 \\ FREQUENCY OF LOCAL COMPLICATIONS AFTER TOTAL HIP ARTHROPLASTY IN PATIENTS WITH RHEUMATIC DISEASES.}

A. Khramov ${ }^{1}$, M. Makarov ${ }^{1}$, S. Makarov ${ }^{1}$, E. Naryshkin ${ }^{1}$, S. Maglevaniy ${ }^{1}{ }^{1}$ V.A. Nasonova Research Institute of Rheumatology, Orthopaedic, Moscow, Russian Federation

Background: Surgical treatment of patients with rheumatic diseases (RD) is associated with an increased risk of complications. It is caused by presence of an inflammatory process, osteoporosis, reduced physical activity, severity of functional impairment, long-term glucocorticoid therapy, biological and disease-modifying antirheumatic drugs. All this provides elongated wound healing period, the development of infectious complications and increased risk of periprosthetic fractures. Objectives: To study a frequency of local complications of total hip arthroplasty (THA) in patients with inflammatory RD and osteoarthritis (OA).

Methods: We analyzed 1591 THA, which were performed to RD patients between 2000 and 2019 years.

Results: We performed 882 arthroplasties in patients with inflammatory RD, which consisted of patients with rheumatoid arthritis (RA), systemic lupus erythematosus (SLE), juvenile rheumatoid arthritis (JRA), ankylosing spondylitis (AS), systemic scleroderma (SSD), and also 709 operations in OA patients. Local complications after THA were $120(7.54 \%)$, of these $83(9.41 \%)$ in patients with inflammatory RD and $37(5.22 \%)$ in OA patients.

We revealed a significantly greater number of complications in patients with inflammatory RD $(p<0.005)$

Conclusion: Inflammatory RD (RA, SLE, JRA, AS, SSD) patients have local complications after THA (9.41\%) 1.8 times more often than OA patients $(5.22 \%)$. It shows that the operative treatment of patients with $\mathrm{RD}$ requires a special approach, management and careful treatment of the bone and surrounding tissues during surgery.

Disclosure of Interests: None declared

DOI: 10.1136/annrheumdis-2020-eular.5244 